\title{
Condensation principles with rates
}

by

W. DICKMEIS and R. J. NESSEL (Aachen)

Abstract. 'l'ho purpose of this papor is to supply tho classical condensation principle of Bunawe-steinhans (1027) with rates. The method of proof consists in the familing gliding hump musthod, but now oquippod with rates. First applications concerning the sharpnoss of (pointwise) orror bounds are given in connection with Fourier purtitul sums, Bornsticin polynomials, Lagrange interpolation, and numerical quadrature.

1. Introduction. Ist $X$. be a Banach space, $Y$ a normed linear space (with norms $\|\cdot\|_{X},\|\cdot\|_{Y}$, respoctively), and let $[X, Y]$ be the space of bounded linear operutors of $X$ into $Y$. Then one version of the classical condensation principle (OT) reads ( $\boldsymbol{N}$ : = set of natural numbers):

OT. Tet $\left\{T_{n, p}\right\}_{n, p \in \mathbf{N}} \subset[X, Y]$. Suppose that for each $n, p \in \mathbf{N}$ there exists $h_{n, 2)} \in X$ such that

$$
\begin{gathered}
\left\|h_{n, p}\right\|_{X} \leqslant 0, \\
\limsup _{n \rightarrow \infty}\left\|T_{n, p} h_{n, p}\right\|_{Y}=\infty .
\end{gathered}
$$

Then there exists $f_{0} \in X$, independent of $n, p \in \mathbf{N}$, such that

$$
\limsup _{n \rightarrow \infty}\left\|T_{n, p} f_{0}\right\|_{Y}=\infty
$$

simultaneously for all $p \in \mathbf{N}$.

Noto that conditions $(1.1,2)$ are equivalent to the fact that for each $p \in \boldsymbol{N}$

$$
\operatorname{limsip}_{n \rightarrow \infty}\left\|T_{n, p}\right\|_{[X, Y]}=\infty
$$

which, in viow of the classical uniform boundedness principle (UBP), is also orjuivalent to the existence of a sequence $\left\{f_{p}\right\}_{p \in N} \subset X$ such that for each $p \in \boldsymbol{N}$

$$
\limsup _{n \rightarrow \infty}\left\|T_{n, p} f_{p}\right\|_{Y}=\infty
$$

Of course, these remarks just reflect alternative, perhaps more familiar versions of the classical $O P$. 
The CP was shown in 1927 by Banach-Steinhaus [2] via X3airo (atiogory arguments; see also the account givon in [1], p. 81 ff, [11.], p. $23 \mathrm{ff}$ in connection with a number of applications. For a proof via the gliding hump method see [20]. Of course, in viow of the implication (1.5) $\because \cdots(1.3)$, the CP is indeed an extension of the U.BP, which just corresponds to the implication $(1.1,2) \Rightarrow(1.5)$, thus delivering the existence of a sequence $\left\{f_{p}\right\}_{p \in N} \subset X$ rather than a singlo element $f_{0} \in X$, independent of $p$, wuch that (1.5), (1.3), respectively, hold true.

Continuing our previous work on UBP's with ratos (cf. [5], [6], [7]), it is the purpose of this paper to develop CP's with ratos in the senso that (1.1-1.3) are equipped with rates. To this end, Soc. 2 first considers the case of countable index familios. The mothod of proof will bo the gliding hump method but now equipped with rates. Following classical work of Orlicz [15], the results of Soc. 2 are then extended in soc. 3 to uncountable (topologized) index families, using category argumontis. Finally, soc. 4 outlines some first illustrating examples concerned with fiourier partial sums, Bernstein polynomials, Lagrange interpolation, and numerical quadrature of indefinite integrals.

\section{Condensation principles with rates for countable index families.} Then the $\mathbb{K}$-functional $(t \geqslant 0)$

$$
K(t, f):=K(t, f ; X, U):=\inf \left\{\|f-g\|_{X}+t|g|_{U} ; g \in U\right\}
$$

serves as an abstract measure of smoothness for tho olomont $f \in X$ (ci: $(4.1,4))$. Let $\omega$ be a continuous function on $[0, \infty)$ with

$$
\begin{array}{ll}
0<\omega(t) \leqslant \omega(s) & (0<t \leqslant s), \\
\omega(t+s) \leqslant \omega(t)+\omega(s) & (s, t \geqslant 0) .
\end{array}
$$

Note that necessarily $\liminf _{t \rightarrow 0+} \omega(t) / t>0$, in fact (cf. [18], p. $96 \mathrm{ff}$ )

$$
0<\omega(s) / s \leqslant 2 \omega(t) / t \quad(0<t \leqslant s) .
$$

Thus, if $\omega(0)=0$, then $\omega$ is an abstract modulus of continuitiy in the usual sense. Consider the intermediate spaces $(t \rightarrow 0+*)$

$$
\begin{aligned}
& X_{\omega}^{0}=\left\{f \in X ; K(t, f)=o_{f}(\omega(t))\right\}, \\
& X_{\omega}=\left\{f \in X ; K(t, f)=O_{f}(\omega(t))\right\},
\end{aligned}
$$

and let $\left\{p_{n, p}\right\}_{n, p \in N}$ denote a double sequeneo of (strictily) positive numbers.
with with

$$
\lim _{n \rightarrow \infty} p_{n, p}=0
$$

for each $p \in \boldsymbol{N}$. Then one has the following vorsion of a OP with largo
O-rates. Let $U \subset X$ be a seminormed linear subspace of $X$ with sominorm $|\cdot|_{U}$.

Turorfim 1. Let $\left\{T_{p}\right\}_{p \in \boldsymbol{N}},\left\{T_{n, p}\right\}_{n, p \in \boldsymbol{N}} \subset[X, X]$ and let $\omega,\left\{\varphi_{n, p}\right\}_{n, p \in \boldsymbol{N}}$ satisfy (2.2), (2.6), respectively. Suppose that for each $n, p \in \boldsymbol{N}$ there exists: $h_{n, p)} \in U$ such that

$$
\begin{gathered}
\left\|h_{n, p}\right\|_{X} \leqslant O_{1}, \\
\left|h_{n, p}\right|_{U} \leqslant O_{2} / \varphi_{n, p}, \\
\limsup _{n \rightarrow \infty \rightarrow \infty}\left\|T_{n, p} h_{n, p}\right\|_{Y}=\infty \quad(p \in \mathbf{N}) .
\end{gathered}
$$

(a) If a satisfies additionally

$$
\lim _{t \rightarrow 0+1} \omega(t) / t=\infty,
$$

then lhere exists $f_{01} \in X_{(1)}^{\prime \prime}$, independent of $p \in \boldsymbol{N}$, such that for each $p \in \mathbf{N}$

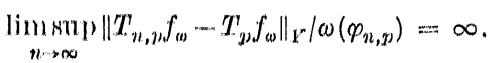

(b) If $\omega(t)=\omega_{1}(t):=t$ (i.e., essentially, one does not have (2.10) (cf. (2.3))), then there exists $\int_{\omega} \in X_{\omega}$, independent of $p \in N$, such that (2.11) holds true $\left(\right.$ for $(1)=\left(\omega_{1}\right)$.

Thus, in any case, there exists an element $f_{\omega}$ as specified above such that for each $p \in \boldsymbol{N}$

$(2.11)^{*} \quad\left\|T_{n, m} f_{\omega}-T_{p} f_{\omega}\right\|_{X} \neq O\left(\omega\left(\varphi_{n, p}\right)\right) \quad(n \rightarrow \infty)$.

Proof. Lot the socuonco $\left\{p_{k}\right\}_{k \in N} \in \boldsymbol{N}$ be given via

$$
p_{1}:=1, \quad p_{k+2}:=\left\{\begin{array}{lll}
p_{k}-1, & \text { if } & p_{k}>1 \\
k+1, & \text { if } & p_{k}=1,
\end{array}\right.
$$

in other words, if $k=2^{l}+m, 0 \leqslant m<2^{l}, m, l \in \boldsymbol{N} \cup\{0\}$, then $p_{k}=2^{l}-m$. It follows that for anch $p \in \boldsymbol{N}$ there are infinitely many $k_{i} \in \boldsymbol{N}$ with $p_{k}=p$. Now one may successively construct sequences $\left\{a_{k}\right\}_{k \in N} \subset(0,1 / 2],\left\{\delta_{k}\right\}_{k \in N}$ $\subset\{0,1\},\left\{n_{k}\right\}_{k e N} \subset N$ with $a_{1}=1 / 2, \delta_{1}=n_{1}=1$ such that for $k \geqslant 2$

$$
0<a_{k} \leqslant a_{k-1} \leqslant 1 / 2
$$

$$
O \text {-rates. }
$$

$$
\text { (i) } \quad n_{k}>n_{k-1}, \quad p_{n_{k,}, p_{k}}<p_{n_{k-1}, p_{k-1}} \text {, }
$$

and in case (a) additionally

$$
\begin{aligned}
& \sum_{j=1}^{k m=1} a_{j}^{j} \omega\left(\varphi_{n_{j}, m_{j}}\right) / \varphi_{n_{j}, p_{j}} \leqslant a_{k}^{l_{k}} \omega\left(\varphi_{n_{k,}, \eta_{k}}\right) / \varphi_{n_{k},}, n_{k}, \\
& \left\|T_{n_{k \cdots 1}, \eta_{k-1}}-T_{p_{k m-1}}\right\|_{[X, Y]} a_{k}^{k} 2 O_{1} \leqslant\left(l_{0}-1\right) / 2,
\end{aligned}
$$

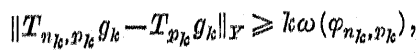

$$
\begin{aligned}
& g_{k}:=\sum_{j=1}^{h} \delta_{j} a_{j}^{j} \omega\left(\varphi_{n_{j}, p_{j}}\right) n_{n_{j}, \eta_{j}} \in U \text {. }
\end{aligned}
$$


Indeed, if the first $k-1$ elements of the sequences, and thus $g_{k, \ldots l} \in U$, aro given, choose $a_{l}$ small enough so that $(2.13,15)$ axe satisliod. Consillor

$$
M M_{k-1}:=\limsup _{n \rightarrow \infty}\left\|T_{n, p_{k}} g_{k-1}-T_{p_{k}} g_{k-1}\right\|_{Y} / \omega\left(p_{n, 2)_{k}}\right) \text {. }
$$

In case $M_{k-1}<$ co thero exists an integor $n_{\%}$ with

$$
\left\|T_{n_{k}, p_{k}} g_{k-1}-T_{p_{k}} g_{k_{i-1}}\right\|_{\boldsymbol{Y}} \leqslant\left(M_{k_{k-1}}+1\right) \omega\left(p_{n_{k}, p_{\eta_{k}}}\right) \text {. }
$$

Moreover, $n_{\%}$ can be chosen so large that $(2.14)$ is satisfied (cf. (2.6, 10)), as well as

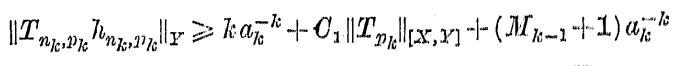

(cf. $(2.9)$ ). Fence, setting $\delta_{k}=1$, the eloment $g_{k}$ is woll-ilofinch and (cf. $(3.7))$

$$
\begin{aligned}
& \left\|T_{n_{k}, p_{k}} g_{k}-T_{p_{k}} g_{k}\right\|_{Y} \geqslant a_{l_{k}}^{k} \omega\left(p_{n_{k}, p_{k}}\right)\left\|T_{n_{k}, p_{k}} h_{n_{k}, \eta_{k} \eta_{k}}\right\|_{Y}-
\end{aligned}
$$

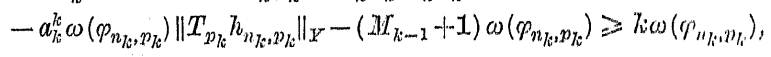

i.e., (2.16) holds true. In case $M_{k-1}=\infty$, condition (2.16) (as well as (2.14)) of course holds true for $g_{k}:=g_{k-1}$, i.e., $\delta_{k}:=0$, with suitablo $\eta_{k}$.

$$
\text { Since } X \text { is complete and (cf. }(2.2,7,13,14) \text { ) }
$$

$$
\sum_{j=1}^{\infty} \delta_{j+1} a_{j}^{j} \omega\left(p_{n_{j}, \nu_{j}}\right)\left\|h_{n_{j}, p_{j}}\right\|_{X} \leqslant \sigma_{1} a_{k+1}^{k+1} \omega\left(p_{n_{k+1}, n_{k+1}}\right) \sum_{j=0}^{\infty} 2^{-j}<\infty,
$$

the element

$$
f_{\omega}:=\sum_{j=1}^{\infty} \delta_{j} a_{j}^{j} \omega\left(\varphi_{n_{j}, x_{j}}\right) h_{n_{j}, n_{j}}
$$

is well-defined in $X$. Moreover, $f_{\omega} \in X_{\omega}^{0}$ and $f_{\omega_{1}} \in X_{\omega_{1}}$, respectively. Indeed, since for each $t \in\left(0, \varphi_{1,1}\right)$ there exists $k$ such that $\varphi_{n_{k+1}, p_{k+1}} \leqslant t<p_{n_{k}, n_{k}}$ (note that $\lim \varphi_{n_{k}, p_{k}}=0$ in view of $(2.6,12,14)$ ), one obtitins ill case (a) by $(2.1,8,13,14$ (ii), 17) and finally $(2.2,3)$ that

$$
\begin{aligned}
& K\left(t, f_{\omega}\right) \leqslant\left\|f_{\omega}-g_{k}\right\|_{X}+t\left|g_{k}\right|_{U} \\
& \leqslant \sum_{j=k+1}^{\infty} \delta_{j} a_{j}^{j} \omega\left(\varphi_{n_{j}, v_{j}}\right)\left\|h_{n_{j}, p_{j}}\right\|_{x}+t \sum_{j=1}^{k} \delta_{j} a_{j}^{j} \omega\left(p_{n_{j}, p_{j}}\right)\left|h_{n_{j}, p_{j}}\right| v
\end{aligned}
$$

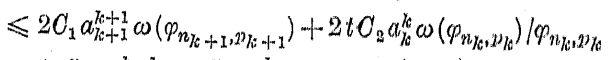

$$
\begin{aligned}
& \leqslant\left(2 O_{1} 2^{-t i-1}+4 O_{2} 2^{-l t}\right) \omega(t)=o(\omega(t)) \text {, }
\end{aligned}
$$

i.e., $f_{\omega} \in X_{\omega}^{0}$. In case (b) one obtains analogously

$$
\begin{aligned}
K\left(t, f_{\omega_{1}}\right) & \leqslant\left\|f_{\omega_{1}}-g_{k}\right\|_{x}+t\left|g_{k}\right|_{U} \\
& \leqslant 2 \sigma_{1} \varphi_{n_{k+1}, p_{k+1}}+t O_{2} \sum_{j=1}^{\infty} a_{j}^{j} \leqslant \theta t,
\end{aligned}
$$

thus $f_{w_{1}} \in X_{\omega_{1}}$. Now (2.1.5-17) deliver

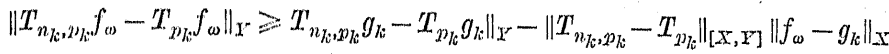

$$
\begin{aligned}
& \geqslant(k / 2) \omega\left(p_{n_{k}, x^{\prime} k_{k}}\right) \text {. }
\end{aligned}
$$

Since for owoh $p \in \boldsymbol{N}$ there are infinitely many $k \in \boldsymbol{N}$ such that $p_{k}=p$ (ef. (2.12)), this completes the proot.

Iut us montion that Thm. 1. (a) substmes the classical CP as a special cuse, namoly for $\omega=\omega_{0}:=1, U=X, T_{p}=0$. Indeed, conditions $(1.1,2)$ and $(2.7,9)$ coincide, whorous $(2.8)$ is then trivially satisfied for any $\left\{p_{n, \eta}\right\}$. Note that now $X_{\omega}^{0}=x$.

Doding with (proper) rates, however, one may even replace large $O$ ratios in I'lum. 1. (a) by small o-ones if the limiting case $\omega_{0}$ (and $\omega_{1}$ ) is excluded.

THwonw. 2. Let $\left\{T_{n, p}\right\}_{n, p \in \mathcal{N}} \subset[X, Y]$, and let $\left\{\varphi_{n, p}\right\}_{n, p \in N}$ satisfy (2.6). Suppose that for each $n, p \in \boldsymbol{N}$ there exists $h_{n, p} \in U$ such that conditions $(2.7,8)$ hold true as well as

$$
\limsup _{n \rightarrow \infty}\left\|T_{n, p} h_{n, p}\right\|_{Y} \geqslant O_{3}>0 .
$$

Then there exists a constant $O_{4}>0$ such that for each modulus $\omega$ satisfying (2.2) as woll as (2.10) and

$$
\lim _{t \rightarrow 0+0^{+}} \omega(t)=\omega(0)=0
$$

there exists $f_{\omega} \in X_{\omega}$, independent of $p \in \boldsymbol{N}$, such that for each $p \in \mathbf{N}$

$$
\limsup _{n \rightarrow \infty}\left\|T_{n, p} f_{\omega}\right\|_{Y} / \omega\left(\varphi_{n, p}\right) \geqslant 0_{4} .
$$

In particular,

$$
(2.20)^{*} \quad\left\|T_{n, p} f_{\omega}\right\|_{X} \neq o\left(\omega\left(\varphi_{n, p}\right)\right) \quad(n \rightarrow \infty) .
$$

Proof. Procooding as in the proof of $\mathrm{Thm} .1$ (a), lot the sequence $\left\{p_{t}\right\}_{k e N}$ be given by $(2.12)$. Then one may construct sequences $\left\{n_{k}\right\}_{k \in N}$ $\subset \boldsymbol{N},\left\{\delta_{k}\right\}_{k \in N} \subset\{0,1\}$ with $n_{1}=\delta_{1}=1$ satisfying for $k \geqslant 2$ conditions (2.14) (ot' conxse, (ii) without any $a_{j}^{\prime}$ 's) as woll as

$$
\begin{aligned}
& \omega\left(p_{n_{k}, y_{k}}\right) \leqslant(1 / 2) \omega\left(\varphi_{n_{k-1}, y_{j_{k-1}}}\right), \\
& \left\|T_{n_{k-1}, n_{k-1}}\right\|_{[x, x]} \leqslant\left(O_{3} / 200_{i}\right) \omega\left(\varphi_{n_{k-1}, p_{k-1}}\right) / \omega\left(\varphi_{n_{k}, \eta_{k_{k}}}\right) \text {, }
\end{aligned}
$$

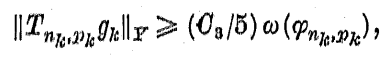

$$
\begin{aligned}
& g_{k}:=\sum_{j=1}^{h_{f}} \delta_{j} \omega\left(\varphi_{n_{j}, p_{j}}\right) h_{n_{j}, p_{j}} \in U .
\end{aligned}
$$


Indeed, if the first $k-1$ elements of the sequences, and thus $g_{k-1} \in U$, are given, one may consider

$$
M_{k-1}:=\limsup _{n \rightarrow \infty}\left\|T_{n, p_{k}} g_{k-1}\right\|_{Y} / \omega\left(\varphi_{n, p_{k}}\right) \text {. }
$$

such that for each $t \in \mathcal{N}$

If $M_{k-1} \leqslant 2 C_{3} / 5$, one has for all but a finite number of values of $n$

$$
\left\|T_{n, p_{k}} g_{k-1}\right\|_{Y} / \omega\left(\varphi_{n, p_{k}}\right) \leqslant 3 C_{3} / 5 \text {. }
$$

Therefore, setting $\delta_{k}:=1$, wo may choose $n_{k}$ (herowith detormining $h_{n_{k}, p_{l_{c}}}$ and $g_{k_{k}}$ ) large enough to satisfy $(2.14,21,22,24)$ (cf. $\left.(2.19)\right)$ as well as (cf. (2.18))

$$
\left\|T_{n_{k}, n_{\eta_{k}}} h_{n_{k}, n_{k}}\right\|_{Y r} \geqslant 4 . O_{3} / 5
$$

thus (2.23). If $M_{k-1}>2 O_{3} / 5$, take $\delta_{k}=0$ thus $g_{k}:=g_{k-1}$. Thon, of courso. one may find $n_{k}$ large enough to satisfy $(2.23)$ as well as $(2.14,21,22)$,

Since $X$ is complete and (cf. $(2.7,21)$ )

$$
\sum_{j=k_{c+1}}^{\infty} \delta_{j} \omega\left(\varphi_{n_{j}, p_{j}}\right)\left\|h_{n_{j}, p_{j}}\right\|_{X} \leqslant \theta_{1} \omega\left(p_{n_{k+1}, p_{k+1}}\right) \sum_{j=0}^{\infty} 2^{-j}<\infty,
$$

the element

$$
f_{\omega}:=\sum_{j=1}^{\infty} \delta_{j} \omega\left(\varphi_{n_{j}, p_{j}}\right) h_{n_{j}, p_{j}}
$$

is well-defined in $X$. Moreover, $f_{\omega} \in X_{\omega}$. Indeed, choosing $t, k$ as in the proof of Thm. 1, one analogously obtains (cf. (2.14 (ii), 25))

$$
\begin{aligned}
K\left(t, f_{\omega}\right) & \leqslant\left\|f_{\omega}-g_{k}\right\|_{X}+t\left|g_{k}\right|_{U} \\
& \leqslant 2 C_{1} \omega\left(\varphi_{n_{k+1}, p_{k+1}}\right)+2 C_{2} t \omega\left(p_{n_{k}, p_{k}}\right) / \varphi_{n_{k}, p_{k}} \leqslant\left(2 O_{1}+4 C_{2}\right) \omega(t) .
\end{aligned}
$$

Now by $(2.22,23,25)$

$$
\begin{aligned}
\left\|T_{n_{k}, p_{k}} f_{\omega}\right\|_{Y} & \geqslant\left\|T_{n_{k}, p_{k}} g_{k}\right\|_{Y}-\left\|T_{n_{k}, p_{k}}\right\|_{[X, Y]}\left\|f_{\omega}-g_{k}\right\|_{X} \\
& \geqslant\left[C_{3} / 5-C_{3} / 10\right] \omega\left(\varphi_{n_{k}, p_{k}}\right) .
\end{aligned}
$$

In view of (2.12) this proves (2.20) with $C_{4}=C_{3} / 10>0$.

Note that the limiting cases $\omega=\omega_{0}, \omega_{1}$ hare to be excluded in Thm. 2 . In fact, this remark already applies to the particular situation of the UBR? with rates (case $T_{n, p}=T_{n}$, cf. [5], [7]).

3. Condensation for uncountable index families. Whilo the proceding section was concerned with doublo sequences of operators wo now want to give a first contribution to the case whon $p$ varies over an uncountablo index set $\mathscr{A}$. To this end we shall use the following lommas.

LEMra 1. Let $\mathscr{A}$ denote a topological space of second category and $\mathscr{N} \subset \mathscr{A}$ a dense subset. Let $\left\{g_{n}\right\}_{n \in \mathbb{N}}$ be a sequence of continuous functions on $\mathscr{A}$
Then the set $(\mathscr{N} \subset \mathscr{B} \subset \mathscr{A})$

$$
\limsup _{n \rightarrow \infty} g_{n}(t)=\infty .
$$

$$
\mathscr{B}:=\left\{t \in \mathscr{A} ; \limsup _{n \rightarrow \infty} g_{n}(t)=\infty\right\}
$$

is of second category in $\mathscr{A}$

Lewma 2. Let $\mathscr{A}, \mathcal{N},\left\{g_{n}\right\}_{n e N}$ satisfy the hypotheses of La. 1 with (3.1) replaced by

$$
\limsup _{n \rightarrow \infty} g_{n}(t) \geqslant c_{0}>0
$$

Then the $\operatorname{set}\left(\mathcal{N} \subset \mathscr{B}^{\prime} \subset \mathscr{A}\right)$

$$
\mathscr{B}^{\prime}:=\left\{t \in \mathscr{A} ; \limsup _{n \rightarrow \infty} g_{n}(t) \geqslant c_{0}\right\}
$$

is of second category in $\mathscr{A}$.

Proofs. For La. 1. seo Orlicz [15]. In fact, the proof of La. 2 is just a copy of that givon in [15], but included here for the sake of completeness. sinee $g_{n}$ is continuous on $\mathscr{A}$, the sets $(m, n \in \boldsymbol{N})$

$$
\mathscr{S}_{n, m}:=\left\{t \in \mathscr{A} ; g_{n}(t) \leqslant c_{0}(1-1 / m)\right\}
$$
are closed. Thus also the intersoctions $\mathscr{F}_{k, m}:=\bigcap_{n \geqslant l} \mathscr{P}_{n, m}$ are closed in $\mathscr{A}$.
Now observo that

$$
\mathscr{A} \backslash \mathscr{B}^{\prime}=\left\{t \in \mathscr{A} ; \limsup _{n \rightarrow \infty} g_{n}(t)<c_{0}\right\}=\bigcup_{m \in \boldsymbol{N}} \bigcup_{k \in \boldsymbol{N}} \mathscr{F}_{k, m} .
$$

Moreover, oach set $\mathscr{F}_{k, m}$ is nowhere dense in $\mathscr{A}$. Indeed, suppose there is a sot $\mathscr{F}_{k_{0}, m_{0}}$ with an inner point $t_{0}$ in its closure. Then $\mathscr{F}_{k_{0}, m_{0}}$ contains an open neighbourhood $\mathscr{V}_{0}$ since $\mathscr{F}_{k_{0}, m_{0}}$ is closed. Of course, $\mathscr{V}_{0} \subset \mathscr{A} \backslash \mathscr{B}$, but tho density of $\mathscr{N}$ in $\mathscr{A}$ also implies $\mathscr{V}_{0} \cap \mathcal{N} \neq \varnothing$ and thus $\mathscr{V}_{0} \cap \mathscr{B}^{\prime} \neq \varnothing$, a contradiction. Honco $\mathscr{A} \backslash \mathscr{B} \mathscr{B}^{\prime}$ is of first category. Since $\left.\mathscr{A}=\mathscr{B} \mathscr{B}^{\prime} \cup \mathscr{A} \backslash \mathscr{B} \mathscr{B}^{\prime}\right)$ Was askumod to bo of second eategory, $\mathscr{B}$ ' must be of socond category, too.

Noto that luk. "1, 2 do not stato that $\mathscr{B}$ (or $\mathscr{B} B^{\prime}$ ) is equal to $\mathscr{A}$. In fact, this cannot ho trues in genoral as already montioned in [.15] (see also Sec. 4.1.).

Of courno, Lath. 1, 2 may now be used to derive extensions of the results of Sos. 2 to fumilies of operators depending on an uncountablo index $p$. Rather than to formulato a most general theorem, let us just state one typical version.

Ooroluary 1. Let denote an interval of $\boldsymbol{R}(:=$ the real line with the natural topology), and let $\mathcal{N}$ be the set of rational numbers in $\mathscr{A}$. For $n \in N, t \in \mathscr{A}$ let $T_{n, t} \in[X, Y]$, and let $p_{n, t}$ satisfy (2.6) such that for all 
$n \in \mathbf{N}, t \in \mathbb{N}$ conditions $(2.7,8,18)$ hold true. Suppose that

$$
g_{n}(t):=\left\|T_{n, t} f\right\|_{X} / \omega\left(\varphi_{n, t}\right)
$$

is a continuous function on $\mathscr{A}$ for each $f \in X$. Then there exists a constant $C_{4}>0$ such that for each modulus $\omega$ satisfying $(2.2,10,19)$ there exists $f_{(1)} \in X_{w}$, independent of $t$, with

$$
\limsup _{n \rightarrow \infty}\left\|T_{n, t} f_{\omega}\right\|_{T^{-}} / \omega\left(p_{n, t}\right) \geqslant O_{4}
$$

simultaneously for all t in a set of second oategory in $\mathscr{A}$, containing $\mathcal{N}$.

\section{Applications.}

4.1. Fourier partial sums. Let $C_{3}$ denote the space of contimnous, $2 \pi$-periodic functions with the usual sup-norm and $C_{2 \pi}^{(1)}$ the subspace of continuously differentiable functions (with seminorm $\left\|f^{\prime}\right\|_{0}$ ). Tho colresponding $K$-functional turns out to be equivalent to the ustal molulus of continuity of functions, i.e., if

$$
\omega(t, f):=\sup _{|h| \leqslant t}\|f(x+h)-f(x)\|_{C},
$$

then there exist constants $c_{1}, c_{2}>0$, independent of $f \in O_{2 \pi}, t \geqslant 0$, such that (cf. [4], p. $192 \mathrm{f}$ )

$$
c_{1} \omega(t, f) \leqslant K\left(t, f ; O_{2 \pi}, O_{2 \pi}^{(1)}\right) \leqslant c_{2} \omega(t, f) .
$$

For the Fourier partial sums

$$
\begin{aligned}
\left(S_{n} f\right)(x): & =\sum_{|k| \leqslant n} f^{\wedge}(k) e^{i k x}=\frac{1}{2 \pi} \int_{-\pi}^{\pi} f(x-u) D_{n}(u) d u, \\
f^{\wedge}(k): & =\frac{1}{2 \pi} \int_{-\pi}^{\pi} f(u) e^{-i k u} d u, \quad D_{n}(u):=\sum_{|k|<\varepsilon, n} e^{i k u,}
\end{aligned}
$$

one has the well-known (uniform) direct estimate

$$
\left|\left(S_{n} f\right)(x)-f(x)\right| \leqslant \theta \omega\left(n^{-1}, f\right) \log n \quad(\infty \in \boldsymbol{R}) .
$$

Then Thm. 2 and La. 2 (or Cor. 1) give

COROLLATR 2. For each modulus w satisfying $(2.2,10,19)$ there exists $f_{\omega} \in C_{2 \pi}$ such that

but on the other hand

$$
\omega\left(t, f_{\omega}\right)=O(\omega(t)) \quad(t \rightarrow 0+),
$$

$$
\limsup _{n \rightarrow \infty}\left|\left(S_{n} f_{\omega}\right)(x)-f_{\omega}(x)\right| / \omega(1 / n) \log n \geqslant c_{0}>0
$$

simultaneously for all $x$ in a dense set of seoond category in $\boldsymbol{R}$.
Troof. Tost we first cheok the conditions of Thm. 2 for the spaces $X=C_{2 \pi}, X=C\left(:=\right.$ tho complex plane), $U=C_{2 \pi}^{(1)}$, and the bounded linctre functionals $T_{n, x} f:=\left[\left(S_{n} f\right)(x)-f(x)\right] / \log n$. Since for the functionals $S_{n, r} f:=\left(S_{n} f\right)(x)$ ono has $\left\|S_{n, x}\right\|_{\left[O_{2 \pi},\right.},=\left\|D_{n]}\right\|_{1}>c \log n$, independent of $x$, thare aro demontis $f_{n, x} \in G_{z \pi}$ with $\left\|f_{n, x}\right\|_{d}=1$ and $\left[S_{n, x} f_{n, x} \mid \geqslant e^{\prime} \log n\right.$. Now choose $h_{n, n}=V_{n} f_{n, x}$, whore $V_{n}:=(1 / n) \sum_{n=m+n+1}^{2 n} S_{k}$ aro the standard delity mathms of do Tat Vallog Poussin. Thon $h_{n, x}$ is a trigonometric polynomial of dogreo $2 n$ satisfying $(2.7,8)$ with $\varphi_{n, x}=1 / n$, independent of $x$, als a conserpuenes of the classical Bornstiein inequality. Condition (2.18) is fultillod since for sufficiontly lasgen

$$
\begin{aligned}
\left|I_{n, x} h_{n, x}\right| & =\left|S_{n, x,} V_{n} f_{n, x}-\left(V_{n} f_{n, x}\right)(x)\right| / \log n \\
& \geqslant\left[\left|S_{n, x} f_{n, x}\right|-\sigma_{1}\right] \log n \geqslant c^{\prime \prime}>0
\end{aligned}
$$

Thus an application of Thm. 2 in connection with (4.1) gives the existence of $f_{w}$ satisfying $(4.2,3)$ but only for countable many $x \in \boldsymbol{R}$, e.g., for rational $x$. Since $\left|T_{n, x} f_{w}\right|$ is a continuous function of $x \in \boldsymbol{R}$, application of La. 2 states that (4.3) moreover holds true on a dense set of second category in $\boldsymbol{R}$.

It is interosting to consider the case $\omega(t) \sim(\log (1 / t))^{-1}$. Thon (4.3) stinties that $\limsup _{n \rightarrow \infty}\left|\left(S_{n} f_{m}\right)(x)-f_{(n)}(x)\right| \geqslant c_{0}>0$ on a dense set of second mitegry, while the fimous theorem of Carleson states the convergence alnosti overy where on $\boldsymbol{R}$. This fact does not only show (as already mentioned in Soc.3) that in genoral $\mathscr{B}, \mathscr{B}^{\prime} \neq \mathscr{A}$ in La. 1, 2, but that oven a $\mathrm{OP}^{2}$ with ratos is not possible for an arbitrary (second) index sot without adlitional แลs

In comnoction with the development of the various kinds of UBP's and OP's, the Fourier partial sums have always been used as the test for the applicability of the functional analytical principles under consideration. So there is a lot of background material. Rather than to be complete, let nis only montion that tho present treatmont should be compared with the classical onos, givon in [2] without rates and in [13] with rates, but without condensation. On the other hand, there are even results on the (nom) convorgenco with ratios almost overywhere (seo [16], [17]), which. of comres annot be covered by the present abstract approach.

4.2. Bernstein polynomials. Let $G[0,1]$ denote the space of continuous. functions on $[0,1]$ with the natal sup-norm and $\sigma^{(2)}[0,1]$ the subspace of twico continuously difforontiablo functions (with seminom $\left\|f^{\prime \prime}\right\|_{C}$ ). Agrin tho corresponding $K$-functional is equipalent to the usual second modulus of rontinuity of functions, i.o., if

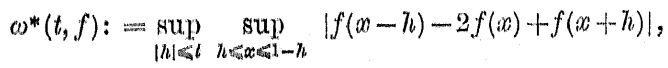


there exist constants $c_{1}, c_{2}>0$ such that

$$
c_{1} \omega^{*}(t, f) \leqslant K\left(t^{2}, f ; O[0,1], O^{(2)}[0,1]\right) \leqslant c_{2} \omega^{*}(t, f) .
$$

For the Bernstein polynomials $(x \in[0,1], n \in \boldsymbol{N})$

$$
B_{n}(f ; x):=\sum_{k=0}^{n}\left(\begin{array}{l}
n \\
k_{k}
\end{array}\right)(1-x)^{n-k} x^{k} f\left(k_{k} / n\right)
$$

one has the well-known direct ostimate (cf. [3])

$$
\left|B_{n}(f ; x)-f(x)\right| \leqslant O \omega^{*}\left(\varphi_{n, x}^{1 / 2}, f\right),
$$

where $\varphi_{n, x}:=x(1-x) / n$. Now an application of the general results of the previous sections deliver

Corollary 3. For each modulus a satisfying (2.2, 1.0, 19) there .exists $f_{\omega} \in O[0,1]$ such that

$$
\omega^{*}\left(t, f_{\omega}\right)=O\left(\omega\left(t^{2}\right)\right) \quad(t \rightarrow 0+-)
$$

but on the other hand

$$
\limsup _{n \rightarrow \infty}\left|B_{n}\left(f_{\omega} ; x\right)-f_{\omega}(x)\right| \mid \omega\left(p_{n, x}\right) \geqslant c_{0}>0
$$

simultaneously for all $x$ in, a dense set of second category in $[0,1]$.

Pro of. Wo check the conditions of Thm. 2 for the spaces $X=(t[0,1]$, $Y=\boldsymbol{R}, U=C^{(2)}[0,1]$, and the bounded linear functionals $T_{u, x} f$ $:=B_{n}(f ; x)-f(x)$. For $x \in(0,1)$ consider the functions $\left(\xi_{n, x} \in[0,1]\right)$

$$
\begin{aligned}
h_{n, x}(u) & =2 \sin ^{2}\left((x-u) / 2 p_{n, x}^{1 / 2}\right)=1-\cos \left((x-u) / \varphi_{n, x}^{1 / 2}\right) \\
& =(x-u)^{2} / 2 \varphi_{n, x}-(1 / 4 !)(x-u)^{4} h_{n, x}^{(\mathrm{IV})}\left(\xi_{n, x}\right) .
\end{aligned}
$$

Of course, $h_{n, x}$ satisfies $(2.7,8)$ (with $\varphi_{n, x}$ ). Moreover, $(2.18)$ follows immediately, since in view of the positivity of the Bernstein polynomials

$$
\begin{aligned}
\left|B_{n}\left(h_{n, x} ; x\right)-h_{n, x}(x)\right| & =B_{n}\left(h_{n, x} ; x\right) \\
& \geqslant\left(1 / 2 \varphi_{n, x}\right) B_{n}\left((x-u)^{2} ; x\right)-(1 / 4 !) \varphi_{n, x}^{-2} B_{n}\left((x-u)^{4} ; x\right) \\
& =1 / 2-(1 / 4 !)\{3+1 / n x(1-x)-6 / n\} \geqslant 1 / 3,
\end{aligned}
$$

if $n \geqslant 1 / x(1-x)$. Thus Thm. 2 delivers the existence of an olomont $f_{\omega} \in O[0,1]$ such that $(4.5,6)$ hold true for all rationtl $\left.x\right) \in(0,1)$. In viosw of the continuity of $\varphi_{n, x}^{-1}$ on $(0,1)$ an application of lat. 2 complotes the proof.

Let us montion that for $\omega(t)=t^{\beta}, 0<\beta \leqslant 1$, ovon a Bornstein-typo inverse theorem is valid: It states that

$$
\left|B_{n}(f ; x)-f(x)\right| \leqslant C(x(1-x) / n)^{\beta} \Rightarrow \omega^{*}(t, f)=O\left(t^{2 \beta}\right) .
$$

For details concerning this result seo [3], [14].
4.3. Lagrange interpolation. Let $L_{n} f$ denote the Lagrange interpolation polynomials for $f \in O[0,1]$ with respect to the triangular matrix of knots $\left\{0 \leqslant x_{1 n}<x_{2 n}<\ldots<x_{n n} \leqslant 1\right\}_{n \in N}$, i.e.,

$$
x_{n}(f ; x):=\sum_{j=\pi 1}^{n} f\left(x_{j n}\right) l_{j n}(x), \quad l_{j n}(x):=\prod_{\substack{k=1 \\ k: k j}}^{n} \frac{x-x_{k n}}{x_{j n}-x_{k n}} \quad(0 \leqslant x \leqslant 1) .
$$

Sotting

$$
\lambda_{n}(x):=\sum_{j=1}^{n}\left|l_{j n}(x)\right|, \quad \lambda_{n}:=\max _{0 \rightarrow x_{i} 1} \lambda_{n}(x),
$$

one has for almosti overy $x \in[0,1]$ (c. [8])

$$
\limsup _{n \rightarrow \infty} \lambda_{n}(x)=\infty \text {. }
$$

Thus for every langrange interpolation process the following divergence properties hold true.

Conordury 4. Ifor each modulus w satisfying $(2.2,10)$ there exists $f_{\omega} \in O[0,1]$ such that

$$
(4.8(a)) \quad \omega^{*}\left(t, f_{\omega}\right)=o\left(\omega\left(t^{2}\right)\right) \quad(t \rightarrow 0+)
$$

as well as

$$
\limsup _{n \rightarrow \infty}\left|L_{n}\left(f_{\omega} ; x\right)-f_{\omega}(x)\right| / \omega\left(\left(n^{2} \lambda_{n}\right)^{-2}\right)=\infty
$$

simultameously for all $x$ in a dense set of second category in $[0,1]$. For $\omega=\omega_{1}$ condition (4.9) holds true for a function $f_{\omega} \in O[0,1]$ such that

$$
\omega^{*}\left(t, f_{\omega}\right)=O\left(t^{2}\right) \quad(t \rightarrow 0+) .
$$

Proof. We first check the conditions of Thm. 1 for the spaces $X$ $=O[0,1], Y=\boldsymbol{R}, U=\sigma^{(2)}[0,1]$, and the bounded linear functionals $T_{n, x} f:=L_{n}(f ; x), T_{x} f=f(x)$, where $x$ varies over a dense, countable set $\mathscr{N} \subset[0,1]$ of indices for which (4.7) holds true. Since $\lambda_{n}(x)$ $=\left\|T_{n, x}\right\|_{[\alpha, \boldsymbol{R}]}$, there exist functions $f_{n, x} \in O[0,1]$ such that $\left\|f_{n, x}\right\|_{\sigma}$ $\leqslant 1$ and $\limsup \left|L_{n}\left(f_{n, x} ; x\right)\right|=\infty$ for each $x \in \mathscr{N}$. Ohoosing now

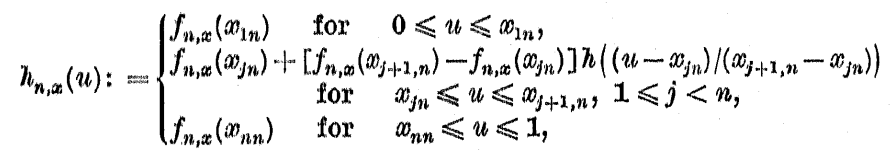

where $h$ denotes some infinitely often differentiable function on $\boldsymbol{R}$ with

$$
h(u)\left\{\begin{array}{lll}
=0 & \text { for } & u \leqslant 0 \\
\epsilon(0,1) & \text { for } & 0<u<1 \\
=1 & \text { for } & u \geqslant 1
\end{array}\right.
$$


one obtains $(2.7-9)$ with $p_{n, x}=1 /\left(n^{2} \cdot \lambda_{n}\right)^{2}$. In fact,

$$
\left\|h_{n, x}\right\|_{C} \leqslant\left\|f_{n, x}\right\|_{C} \leqslant 1, \quad,\left\|h_{n, x}^{i \prime \prime}\right\|_{C} \leqslant 2\left\|h^{\prime \prime}\right\| / d_{n}^{2}
$$

upon using (cf. [9])

$$
d_{n}:=\min _{1 \leqslant j<n}\left(x_{j+1, n}-x_{j n}\right)>1 / n^{2} \lambda_{n}
$$

Moreover, $\limsup \left|T_{n, x} h_{n, x}\right|=\infty$ for each $x \in \mathscr{N}$ sinco $I_{n}\left(h_{n, m} ; u\right)=$ $I_{n}\left(f_{n, x} ; u\right)$. Thus an application of Thm. 1 (a), (b) dolivors the existionec of $f_{\omega}$ satisfying $(4.8(\mathrm{a}),(\mathrm{b}))$ and $(4.9)$ for all $x \in \mathcal{N}$. Since, morcover,

$$
\left|L_{n}\left(f_{\omega} ; x\right)-f_{\omega}(x)\right| / \omega\left(\left(n^{2} \lambda_{n}\right)^{-2}\right)
$$

is a continuous function of $x$ on $\mathscr{A}:=[0,1]$, La. 1 provos $(4.9)$ complotoly.

For a result stating the existence of functions $f_{(n, r}$, deponding upon the individual $x \in[0,1]$ (cf. (1.5)), see [12] (and also [7] for the troatment within the frame of general UBP's with rates). On the other hand, it was shown in [10] (see also [19]) that there exists a continuous function $f_{0}$ such. that $\lim \sup \left|L_{n}\left(f_{0} ; x\right)\right|=\infty$ even for almost all $x$. Of course, results of this type are again beyond the scope of our functional analytical approach :

4.4. Numerical quadrature. For $f \in O[0,1], x \in[0,1]$ consider the trapezoidal rule for the approximate integration of the indefinitio integral $\int_{0}^{x} f(u) d u$, i.e.,

$$
Q_{n, x} f:=h\left\{f(0) / 2+\sum_{i=1}^{n-1} f(k h)+f(x) / 2\right\}, \quad h=x / n .
$$

Then one has the well-known direct estimate $(f \in O[0,1], x \in[0,1])$

$$
\left|Q_{n, x} f-\int_{0}^{x} f(u) d u\right| \leqslant C x \omega^{*}(x / n, f) .
$$

An application of the general results of Sec. 2, 3 now shows that this estimate is indeed sharp in the following senso:

Conoulary 5. For each modulus w satisfying $(2.2,10,1.9)$ there eirists $f_{\omega} \in O[0,1]$ such that

$$
\omega^{*}\left(t, f_{\omega}\right)=O\left(\omega\left(t^{2}\right)\right) \quad(t \rightarrow 0+-),
$$

but on the other hand

$$
\left.\limsup _{n \rightarrow \infty}\left|Q_{n, x} f_{\omega}-\int_{0}^{x} f_{\omega}(u) d u\right||x \omega|(x / n)^{2}\right) \geqslant c>0
$$

simultaneousty for all $x$ in a dense set of second category in $[0,1]$.
Troof. For the spaces $X=C[0,1], X=\boldsymbol{R}, U=O^{(2)}[0,1]$, and the bounded limear functionals $T_{n, x} f:=x^{-1}\left[Q_{n, x} f-\int_{0}^{x} f(u) d u\right], x \in(0,1]$, Thm. 2 dolivers $(4.10,11)$ for all rational $x \in(0,1]$ so that La. 2 completes the argumenti. Tndoed, the functions $h_{n, x}(u)=\sin ^{2}(2 \pi \eta u / x)$ satisfy $(2.7$, 8) with $T_{n}=(x / n)^{2}$ as well as (2.18) since

$$
\begin{aligned}
\left|T_{n, w} h_{n, x}\right| & =x^{\cdots-1} \int_{0}^{x} \sin ^{2}(2 \pi n u / x) d u=n^{-1} \int_{0}^{n} \sin ^{2}(2 \pi u) d u \\
& =\int_{0}^{1} \sin ^{2}(2 \pi u) d u=1 / 2 .
\end{aligned}
$$

Note that $h_{n, x}$ is chosen in such a way that it is positive and vanishes on the knotis of the rule.

Acknowledgments. The contribution of W. Dickmeis was supported in perti by tho Deutscho Forschungsgemeinschaft, Grant No. Ne 171/5-1.

\section{References}

[1.] S. Banach, Théorie des opérations linéaires, Chelsea Publ., Now York 1955 (Orig. od. Warsaw, 1932).

[2] S. Banach, Ir. Steinhaus, Sur le principe de la condensation de singularités, Jiund. Math. 9 (1927), 50-61.

[3] II. Torons, G. G. Lorontz, Inverse theorems for Bernstein polynomials, Indiana Univ, Math. J. 21 (1972), 693-708.

[4] I. I. Butzor, H. Berens, Semi-Groups of Operators and Approximation, Springer, Berlin 1967.

[5] W. Dickmeis, R. J. Nessel, A unified approach to certain counterexamples in approximation theory in connection with a uniform boundedness principle with rates, J. Approximation Theory 31 (1981), 161-174.

[0] -, - -1 uniform boundedness prinoiple with rates and an application to linear processes, in: Finotional Analysis and Approximation (Proc. Conf. Oberwolfach 1980, Eds. P. T. Butzer, E. Görlich, B. Sz.-Nagy), ISNM 60, Birkhäuser, Basel $1981,311-322$.

[7] ...., - On uniform boundedness prinoiples and Banaoh-Steinhaus theorems with rales, Numer. Hunot. Anal. Optim. 3 (1981), 19-52.

[8] T. Erd $8 \mathrm{~s}$, Problems and results on the theory of interpolation I, Acti Math. Acad. Sci. Iungar. 9 (1958), 381-388.

[9] I'. Frdös, P. Turan, On the rale of the Lebesgue functions in the theory of the Iagrange interpolation, ibid. 6 (1955), 47-65.

[10] 1. Erdös, P. O. H. Vórtesi, On the almost everywhere divergenoe of Lagrange interpolatory polynomials for arbitrary systems of nodes, ibid. 36 (1980), 71-89.

[11] S. Kaczmarz, H. Stoinhaus, Thoorio der Orthogonalreihen, Chelsea Publ, Now York 1951 (Orig. ed. Warsaw, 1935).

[12] O. Kis, P. O. H. Vórtesi, On certain linear operators $I$, Acta Math. Acad. Sci. IIuugar. 22 (1.971), 65-71. 
[13] H. Lebesgue, Sur la représentation trigonométrique approchée des fonotions satisfaisant $a$ une condition de Lipschitz, Bull. Soc. Math. France 38 (1910), 184-210.

[14] G. G. Lorentz, Inequalities and the saturation of Bernstein polynomials, in: On Approximation Theory (Proc. Conf. Oberwolfach 1963, Eds. P. I. Butzer, J. Korevaar), ISNM 5, Birkhäuser, Basel 1964, 200-207.

[15] W. Orlicz, Über Folgen linearer Operationen, die von einem Parameter abhängen, Studia Matl. 5 (1934), 160-170.

[16] K. I. Oskolkov, An estimate of the rate of approximation of a continuous function and its conjugate by Fourier sums on a set of lotal measure (Russian), Iav. Acad. Nauk SSSR Ser. Mat. 38 (1974), 1393-1407 [Math. USSR-Izv. 8 (1974), 13721386].

[17] - Lebesgue's inequality in a uniform metrio and on a set of full measure (Russian), Mat. Zametki 18 (1975), 515-526 [Math. Notes 18 (1975), 895-902].

[18] A. F. Timan, Theory of Approximation of Functions, Pergamon Pross, Now York 1963.

[19] P. O. H. Vértesi, On the almost everywhere divergence of Lagrange inlerpolation (Complex and trigonometric cases), Acta Math. Acad. Sci. Hungar. (to appear).

[20] K. Zeller, FK-Räume in der Funktionentheorie I, Math. Z. 58 (1953), 288-305.

\section{On the extension of \\ continuous linear maps in function spaces and the splitness of Dolbeaut complexes of \\ holomorphic Banach bundles}

by

NGUYEN VAN KHUE (Warszawa)

Abstract. The paper investigates the extension of continuous linear maps with values in the spaces of sections of coherent analytic sheaves over analytic spaces. It is shown that the space $H^{0}(X, \mathscr{P})$, where $\mathscr{S}$ is a coherent analytic sheaf over a paracompact an alytic space $X$ has the extension property with respect to the class of

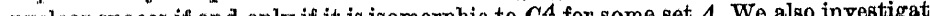
the existence of continuous linear projections of the space $O_{\xi}^{\circ}(R(X))$ onto $C_{\xi}(X)$, where $R(X)$ is the regular part of $X$ and $\xi$ is a holomorphic Banach bundle over $X$. The splitness of Dolbeauti complexes of holomorphic Banach bundles over complex manifolds is considered. We prove that on complex manifolds which are increasing unions of open Stein sots these complexes split only at positive dimensions.

Introduction. In the present paper we consider extensions of continuous linear maps with values in some function spaces of complex analysis and the splitness of Dolbeaut complexes of holomorphic Banach bundles over complex manifolds. These problems have been investigated by soveral authors ([6], [8]). The paper contains three sections.

In $\S 1$ we prove that the space $H^{0}(X, \mathscr{S})$ has the extension property with respect to the class of $s$-nuclear spaces if and only if it is isomorphic to $C^{\wedge}$ tor some set $A$.

Section $\$ 2$ is devoted to the study of the existence of continuous linear projections of $O_{\xi}^{\infty}(R(X))$ onto $\mathcal{O}_{\xi}(X)$. It is shown that when $X$ is Stoin such a projoction exists if and only if $X$ is discrete.

In $\$ 3$ wo investigato the splitness of Dolbeaut complexes of holomorphic Banach bundles over complex manifolds. We prove that on complex manifolds which are increasing unions of open Stein sets these complexes split only at positive dimensions. Let us note that the splitness of Dolbeaut complexes of holomorphic vector bundles over Stein manifolds has been established by Palamodor ([8]). 